\title{
Resection plane-dependent error in computed tomography volumetry of the right hepatic lobe in living liver donors
}

\author{
Heon-Ju Kwon', Kyoung Won Kim², Bohyun Kim³ , So Yeon Kim², Chul Seung Lee ${ }^{2}$, Jeongjin Lee ${ }^{4}$, Gi Won Song ${ }^{5}$, \\ and Sung Gyu Lee ${ }^{5}$ \\ 'Department of Radiology, Kangbuk Samsung Hospital, Sungkyunkwan University School of Medicine, Seoul; '2Department of Radiology, \\ Asan Medical Center, University of Ulsan College of Medicine, Seoul; ${ }^{3}$ Department of Radiology, Ajou University Hospital, Suwon; \\ ${ }^{4}$ School of Computer Science \& Engineering, Soongsil University, Seoul; ${ }^{5}$ Division of Hepatobiliary and Liver Transplantation Surgery, \\ Department of Surgery, Asan Medical Center, University of Ulsan College of Medicine, Seoul, Korea
}

Background/Aims: Computed tomography (CT) hepatic volumetry is currently accepted as the most reliable method for preoperative estimation of graft weight in living donor liver transplantation (LDLT). However, several factors can cause inaccuracies in CT volumetry compared to real graft weight. The purpose of this study was to determine the frequency and degree of resection plane-dependent error in CT volumetry of the right hepatic lobe in LDLT.

Methods: Forty-six living liver donors underwent $\mathrm{CT}$ before donor surgery and on postoperative day 7. Prospective CT volumetry $\left(V_{p}\right)$ was measured via the assumptive hepatectomy plane. Retrospective liver volume $\left(V_{R}\right)$ was measured using the actual plane by comparing preoperative and postoperative CT. Compared with intraoperatively measured weight $(W)$, errors in percentage (\%) $V_{p}$ and $V_{R}$ were evaluated. Plane-dependent error in $V_{p}$ was defined as the absolute difference between $V_{p}$ and $V_{R}$. \% plane-dependent error was defined as follows: $\left|V_{P}-V_{R}\right| / W \cdot 100$.

Results: Mean $\mathrm{V}_{\mathrm{p},} \mathrm{V}_{\mathrm{R}}$, and $\mathrm{W}$ were $761.9 \mathrm{~mL}, 755.0 \mathrm{~mL}$, and $696.9 \mathrm{~g}$. Mean and \% errors in $\mathrm{V}_{\mathrm{p}}$ were $73.3 \mathrm{~mL}$ and $10.7 \%$. Mean error and \% error in $V_{R}$ were $64.4 \mathrm{~mL}$ and $9.3 \%$. Mean plane-dependent error in $\mathrm{V}_{\mathrm{p}}$ was $32.4 \mathrm{~mL}$. Mean \% planedependent error was $4.7 \%$. Plane-dependent error in $V_{p}$ exceeded $10 \%$ of $W$ in approximately $10 \%$ of the subjects in our study.

Conclusions: There was approximately $5 \%$ plane-dependent error in liver $\mathrm{V}_{\mathrm{p}}$ on $\mathrm{CT}$ volumetry. Plane-dependent error in $V_{p}$ exceeded $10 \%$ of $W$ in approximately $10 \%$ of LDLT donors in our study. This error should be considered, especially when CT volumetry is performed by a less experienced operator who is not well acquainted with the donor hepatectomy plane. (Clin Mol Hepatol 2018;24:54-60)

Keywords: Liver transplantation; Living Donors; Volumetric computed tomography; Organ volume; Liver

\section{INTRODUCTION}

Living donor liver transplantation (LDLT) is a widely used option for the treatment of patients with end-stage liver diseases. To meet the large metabolic demand of adult recipients, the right hepatic lobe is preferred rather than the left hepatic lobe as a

\begin{abstract}
Abbreviations:
HAP, hepatic arterial phase; LDLT, living donor liver transplantation; SD, standard deviation; $V P$, venous phase; $V_{p}$, prospective $C T$ volumetry; $V_{R^{\prime}}$ retrospective $C T$ volumetry; $W$, intraoperative weight
\end{abstract}

\author{
Corresponding author : Kyoung Won Kim \\ Department of Radiology, Asan Medical Center, University of Ulsan \\ College of Medicine, 88 Olympic-ro 43-gil, Songpa-gu, Seoul 05505, \\ Korea \\ Tel: +82-2-3010-4385, Fax: +82-2-476-4719 \\ E-mail:kimkw@amc.seoul.kr \\ http://orcid.org/0000-0001-6471-6727
}


Heon-Ju Kwon, et al. Resection plane-dependent error of CT volumetry

graft in adult-to-adult LDLT. ${ }^{1,2}$ In order to use a right hepatic lobe as a graft for LDLT, an exact pre-operative assumption of the graft weight is the essential step, because a 'small-for-size' graft in which the graft-to-recipient weight ratio is less than $0.8-1 \%$ may result in graft malfunction or metabolic insufficiency for the recipient and because over-resection of the donor liver may threaten the donor's safety. ${ }^{3-8}$

Computed tomography (CT) hepatic volumetry is currently accepted as the most reliable method for preoperative assumption of the graft weight for living donor liver transplantation. ${ }^{9-20}$ However, the estimated value from CT volumetry can be deviated from the real graft weight due to several factors, including (a) a difference between the preoperative assumptive and the actual hepatectomy planes, (b) the blood volume contained in the large hepatic vessels, ${ }^{2,9-11}$ and (c) the variable physiological density of the liver graft. ${ }^{12,13}$ It may therefore be worthwhile to determine how much each factor contributes to the error of CT volumetry. In our institution, we routinely obtain CT scans on postoperative day 7 in donors, as well as recipients, for the screening of postoperative complications. Therefore, we assumed that the actual resection planes for the procurement of the right hepatic lobe can be determined using postoperative CT scans in the donor and the recipient as a reference, and thereby the error of CT volumetry due to the difference between the preoperative assumptive and the actual hepatectomy planes can be assessed.

Thus, the purpose of this study was to determine the resection plane-dependent error of CT volumetry for right hepatic lobe in live liver donors.

\section{MATERIALS AND METHODS}

\section{Study population}

This study was approved by our institutional review board (2014-1012). They permitted waiving informed consent from study subjects for this retrospective study.

The study group consisted of 46 live liver donors (mean age, 27.0 years \pm 8.2 ; range, 16 - 46 years; 30 men $[25.5$ years \pm 7.3 ; 16 - 41 years], 16 women [27.0 years \pm 8.2 ; range, 16 - 41 years]) who donated their right hepatic lobe for LDLT from February to March 2014 in our institution. The donors underwent CT scans of the liver as a preoperative work-up for LDLT within 2 months prior to liver procurement. They also underwent CT scans for the screening for surgical complications on postoperative day 7. The recipients (mean age, 50.0 years \pm 8.0 ; range, 31-67 years) consisted of 35 men (50.0 years \pm 8.1 ; range, 31-67 years) and 11 women (50.2 years \pm 8.0 ; range, 31-67 years). Mean body weight was $66.6 \mathrm{~kg} \pm 10.5$ (range, 43.0-89.0 kg). They also underwent CT scans for the screening of surgical complications at least once within postoperative day 7 .

\section{CT protocols}

Preoperative CT scans were obtained with a 64-channel MDCT scanner (Somatom Definition; Siemens Medical Solutions, Erlangen, Germany). The donors fasted for more than 8 hours before CT. CT was performed with a breath hold at the end of expiration. After obtaining unenhanced CT scans, $150 \mathrm{~mL}$ of iopromide (Ultravist 370; Bayer Schering Pharma, Berlin, Germany) was administered at a flow rate of $3 \mathrm{~mL} / \mathrm{sec}$ using a mechanical injector. Biphasic CT scanning was then performed during the hepatic arterial phase (HAP) and the venous phase (VP). By means of a bolus-tracking method (Smart Prep; GE Healthcare or CARE-Bolus; Siemens Medical Solutions), HAP scanning was initiated at 10 seconds after enhancement of the descending aorta reached 100 HU. VP scanning, targeted for good opacification of both the portal and hepatic veins, was initiated 40 seconds after HAP scanning. The scanning parameters for preoperative CT scans were as follows: detector collimation of $0.6 \mathrm{~mm} \times 64$; table pitch of 1 ; gantry rotation time of $0.5 \mathrm{sec} ; 200$ effective $\mathrm{mAs}$; and $100 \mathrm{kVp}$. Images were reconstructed with a $5-\mathrm{mm}$ slice thickness at a 5-mm interval, and were then downloaded to the picture archiving and communication system. In addition, the images were reconstructed at 1-mm section thicknesses at 0.70-mm intervals, and were downloaded to a workstation (Advantage Windows, version 4.6; GE Medical Systems, Chalfont, UK) for 3-dimensional reconstruction of the hepatic vessels. Maximum intensity projection and volume-rendering techniques were used as the standard algorithms.

Postoperative CT scans for recipients were obtained in a similar manner to that of preoperative donor CT scans. Postoperative post-contrast CT scans for donors were obtained using a lowdose protocol (50 effective mAs and $100 \mathrm{kVp}$ ).

\section{CT volumetry}

CT volumetry of the right hepatic lobe was performed twice per donor prospectively (before liver procurement) and retrospectively (3 months after liver procurement). We used the computer-aided 
in-house liver volumetry software for our study, using VP images of preoperative donor CT scans. The overall procedure for semiautomated liver volumetry is as follows. Firstly, modified curvature diffusion filtering was applied to smooth the original CT image while preserving the edge. Secondly, the initial liver outline was detected through the sequential applications of seeded region growing onto level-set speed images, which was generated as a map inversely proportional to the gradient magnitude. ${ }^{21}$ Thirdly, the level-set method performed liver segmentation based on the initially detected liver contour. ${ }^{21}$ Malladi's level-set method was adopted for level-set propagation. ${ }^{22}$ Then, a rolling ball algorithm and the removal of false positives were performed to enhance the liver boundary more accurately. Finally, the radiologist defined a resection plane dividing the liver into right and left hepatic lobes on individually selected slices by inputting 2 end points of the line. When resection lines on at least 2 slices were available, all resection lines on intervening slices in between could be computed utilizing the interpolation technique.

For prospective $C T$ volumetry $\left(V_{\mathrm{p}}\right)$, resection plane for the procurement of the right hepatic lobe was determined by 2 radiologists in consensus after the training session (CS Lee, BH Kim>500 cases, each), based on the right border of the middle hepatic vein superiorly and the Cantlie line (imaginary line between the gallbladder and the inferior vena cava) inferiorly, also using the maximum intensity projections of VP images for reference. Then, the volume of the right hepatic lobe was automatically calculated using a summation-of-area method. ${ }^{14}$

Retrospective $C T$ volumetry $\left(V_{R}\right)$ was performed 3 months after the liver procurement in order to avoid recall bias. The reviewers were blinded to the $V_{p}$ and the intraoperative weight $(W)$ of the right-lobe graft. For reference, postoperative CTs ( $\leq$ postoperative day 7) of donor and recipient were carefully compared to determine the actual resection planes for the procurement of the right hepatic lobe. Also, by applying the surgical resection plane inferred from the postoperative CTs to the CT volumetry, the $V_{R}$ was obtained. The other procedures of volumetry were the same as those of $V_{p}$.

\section{Intraoperative weight measurement}

The right hepatic lobes were procured from each donor by several liver transplantation surgeons. After the procurement of the graft, they shook the excised graft to spill out the remained blood in the large vessels and then measured the weight of the graft on a back table using an electronic laboratory scale. After the weight measurement, they flushed the procured graft via the portal vein with histidine-tryptophan-ketoglutarate solution.

\section{Statistical analysis}

All statistical analyses were performed using commercially available software (Medcalc, Version 16.1; Frank Schoonjans, Mariakeke, Belgium). The significance was defined as $P<0.05$.

Pearson correlation tests were performed to determine the correlation coefficients between $V_{p}$ or $V_{R}$ and $W$.

The following indexes were calculated to measure the deviations of $V_{P}$ and $V_{R}$ from $W$. The errors of $V_{P}$ and $V_{R}$ were defined as the absolute amount of deviation of $V_{p}$ and $V_{R}$ from $W$. The percentage (\%) errors were defined as the errors of $V_{p}$ and $V_{R}$ divided by $\mathrm{W}$ and multiplied by 100 . The plane-dependent error of $V_{p}$ was defined as the absolute amount of difference between $V_{p}$ and $V_{R}$. The $\%$ plane-dependent error of $V_{p}$ was defined as the plane-dependent error of $V_{p}$ divided by $W$ and multiplied by 100 . The following are the Equations:

$$
\begin{aligned}
& \text { Error of } V_{P}=\left|V_{P}-W\right| \\
& \text { Error of } V_{R}=\left|V_{R}-W\right| \\
& \% \text { Error of } V_{P}=\left|V_{P}-W\right| / W \cdot 100 \\
& \% \text { Error of } V_{R}=\left|V_{R}-W\right| / W \cdot 100 \\
& \text { Plane-dependent Error of } V_{P}=\left|V_{P}-V_{R}\right| \\
& \% \text { Plane-dependent Error of } V_{P}=\left|V_{P}-V_{R}\right| / W \cdot 100
\end{aligned}
$$

The difference in graft volume/recipient body-weight ratios using $V_{p}$ and $V_{R}$ were evaluated by paired $t$-test. The difference in the frequency of small-for-size graft (graft volume/recipient bodyweight ratio $<0.8)$ was evaluated.

\section{RESULTS}

In our study, the $V_{p}$ and $V_{R}$ ranged from 469.3 to $1,258.6 \mathrm{~mL}$ and from 462.2 to $1,237.6 \mathrm{~mL}$, respectively. The $\mathrm{W}$ varied between $400.0 \mathrm{~g}$ and $1,050.0 \mathrm{~g}$. The mean \pm standard deviation, median, and 25th and 75th percentiles are summarized with their 95\% confidence intervals in Table 1, and are depicted in box and whisker plots (Fig. 1).

$V_{p}$ strongly correlated with W with $r=0.8898$ (95\% confidence interval, 0.8082 to $0.9378 ; P<0.001)$. The $V_{p}$ showed a deviation of -60.9 to $208.6 \mathrm{~mL}$ from $W$. The $V_{p}$ led to an overestimation compared with W in $39(84.8 \%)$ live liver donors and an underes- 
Heon-Ju Kwon, et al.

Resection plane-dependent error of CT volumetry

Table 1. Summary statistics for semiautomated CT volumetry and intraoperative weight of the right hepatic lobe in 46 living liver donors

\begin{tabular}{lcccc}
\hline Volume or weight & Mean \pm SD & Median & $\mathbf{2 5}^{\text {th }}$ percentile & $\mathbf{7 5}^{\text {th }}$ percentile \\
\hline$V_{p}(\mathrm{~mL})$ & $761.9 \pm 136.6(721.4,802.5)$ & $767.1(731.1,801.3)$ & $681.1(606.6,742.1)$ & $833.7(783.9,899.1)$ \\
$V_{R}(\mathrm{~mL})$ & $755.0 \pm 139.1(713.7,796.3)$ & $765.1(723.7,789.1)$ & $667.5(580.0,752.5)$ & $826.8(780.5,883.9)$ \\
$\mathrm{W}(\mathrm{g})$ & $696.9 \pm 116.4(662.4,731.5)$ & $705.0(650.0,750.0)$ & $630.0(558.7,680.0)$ & $770.0(738.6,800.0)$ \\
\hline
\end{tabular}

Numbers in parentheses are $95 \%$ confidence intervals.

$\mathrm{CT}$, computed tomography; $\mathrm{V}_{\mathrm{P},}$ prospective $\mathrm{CT}$ volumetry; $\mathrm{V}_{\mathrm{R},}$ retrospective $\mathrm{CT}$ volumetry; $\mathrm{W}$, intraoperative weight; $\mathrm{SD}$, standard deviation.

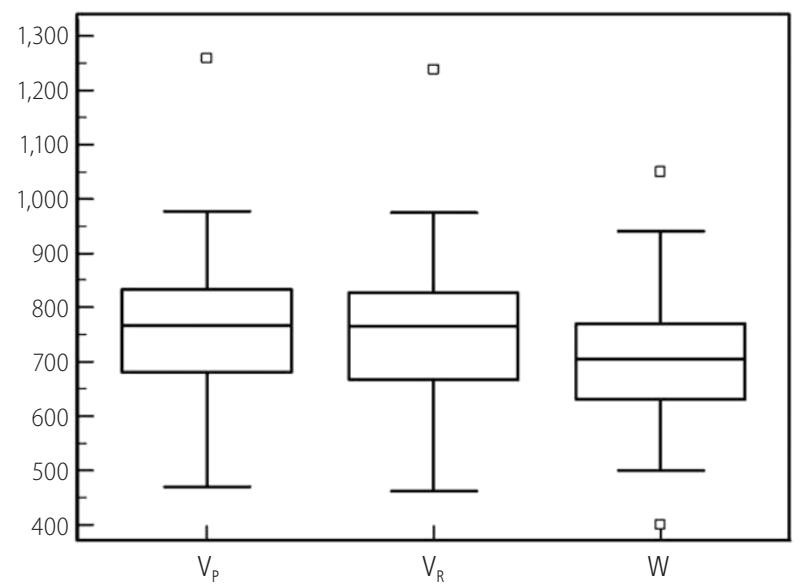

Figure 1. Box and whisker plot of prospective and retrospective computed tomography volumetry and intraoperatively-measured weight of the right hepatic lobe. The central boxes represent the lower to upper quartile (25th to 75 th percentile) values. The middle lines represent the medians. Vertical lines extend from the minimum to the maximum values, excluding "outliers", which are displayed as separate points. The values plotted with a square marker are outliers (smaller than the lower quartile minus 1.5 times the interquartile range or larger than the upper quartile plus 1.5 times the interquartile range [inner fences]]). $V_{p_{r}}$ prospective $C T$ volumetry; $V_{R}$, retrospective $C T$ volumetry; $W$, intraoperative weight.

timation in the other 7 (15.2\%). The error of $V_{p}$ ranged from 0.7 to $208.6 \mathrm{~mL}$, and the $\%$ error of $V_{p}$ was between $0.1 \%$ and $32.3 \%$. $A>10 \%$ error occurred in 23 of 46 donors (50\%).

$V_{R}$ also strongly correlated with $W$ with $r=0.9192$ (95\% confidence interval, 0.8578 to $0.9547 ; P<0.0001)$. The $V_{R}$ showed a deviation of -43.1 to $187.6 \mathrm{~mL}$ from $W$. The $V_{R}$ was overestimated compared with $W$ in 38 (82.6\%) and underestimated in 8 (17.4\%) of 46 live liver donors. The error of $V_{R}$ ranged from 0.1 to 187.6 $\mathrm{mL}$, and the mean $\%$ error of $\mathrm{V}_{\mathrm{R}}$ was between $0.01 \%$ and $25.1 \%$. A $>10 \%$ error occurred in $19(41.3 \%)$ subjects.

Compared with $V_{R}, V_{p}$ deviated from -102.1 to $112.6 \mathrm{~mL}$ (Fig. 2). $V_{P}$ was larger than $V_{R}$ in $28(60.9 \%)$, and smaller than $V_{R}$ in the other 18 (39.1\%). The plane-dependent error of $V_{p}$ was from 1.6 to $112.6 \mathrm{~mL}$. The $\%$ plane-dependent error of $V_{p}$ was between $0.2 \%$ and $18.2 \%$. The plane-dependent error exceeded $10 \%$ compared with W in approximately $10 \%(n=5)$ of the subjects in the prospective setting.

The mean \pm standard deviation, median, and 25th and 75th percentiles of error of $V_{P}$ and $V_{R}$, error of $V_{P}$ and $V_{R}$, and the plane-dependent error and $\%$ plane-dependent error of $V_{p}$ are summarized with their 95\% confidence intervals in Table 2.

Graft volume/recipient body-weight ratios according to $V_{p}$ was $1.16 \pm 0.24 \%$ and that according to $V_{R}$ was $1.16 \pm 0.26 \%$, respectively $(P=0.382)$. There was no small-for-size graft according to $V_{P}$ and $V_{R}$.

\section{DISCUSSION}

Among several factors that may cause the error in CT volumetry in potential live liver donors for LDLT, we focused on the difference between the preoperative assumptive and the actual hepatectomy planes for right lobe procurement. This kind of error could be minimized if CT volumetry is performed by an expert operator (either a radiologist or surgeon), who is fully aware of the donor hepatectomy plane and the possible tailoring transection of segment $V$ vein for optimal sharing of the middle hepatic vein. ${ }^{15}$ However, such problems may occur to a larger extent when the procedure is performed by a less experienced operator. There may be some discrepancy between the line drawn through the middle hepatic vein and the margin of the area of right and left hemiliver, which may curve, undulate, or even interdigitate. ${ }^{23}$ However, the frequency and the degree of such resection plane-dependent errors have not been acknowledged in the prospective setting. In our study, $V_{p}$ showed a $10.7 \%$ difference on average compared to $W$. When we calculated the absolute difference of $V_{P}$ from $V_{R}$ (by applying the actual resection plane), the difference corresponded to $4.7 \%$ of $\mathrm{W}$. Therefore, we suggest that CT volumetry in the prospective setting may produce about a $5 \%$ error, attributed to surgical plane mis-registration. The plane-dependent error of $V_{p}$ exceeded $10 \%$ of $\mathrm{W}$ in approximately $10 \%$ of the subjects in our study. 


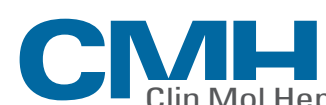

Clin Mol Hepatol

Volume 24 Number1 March 2018
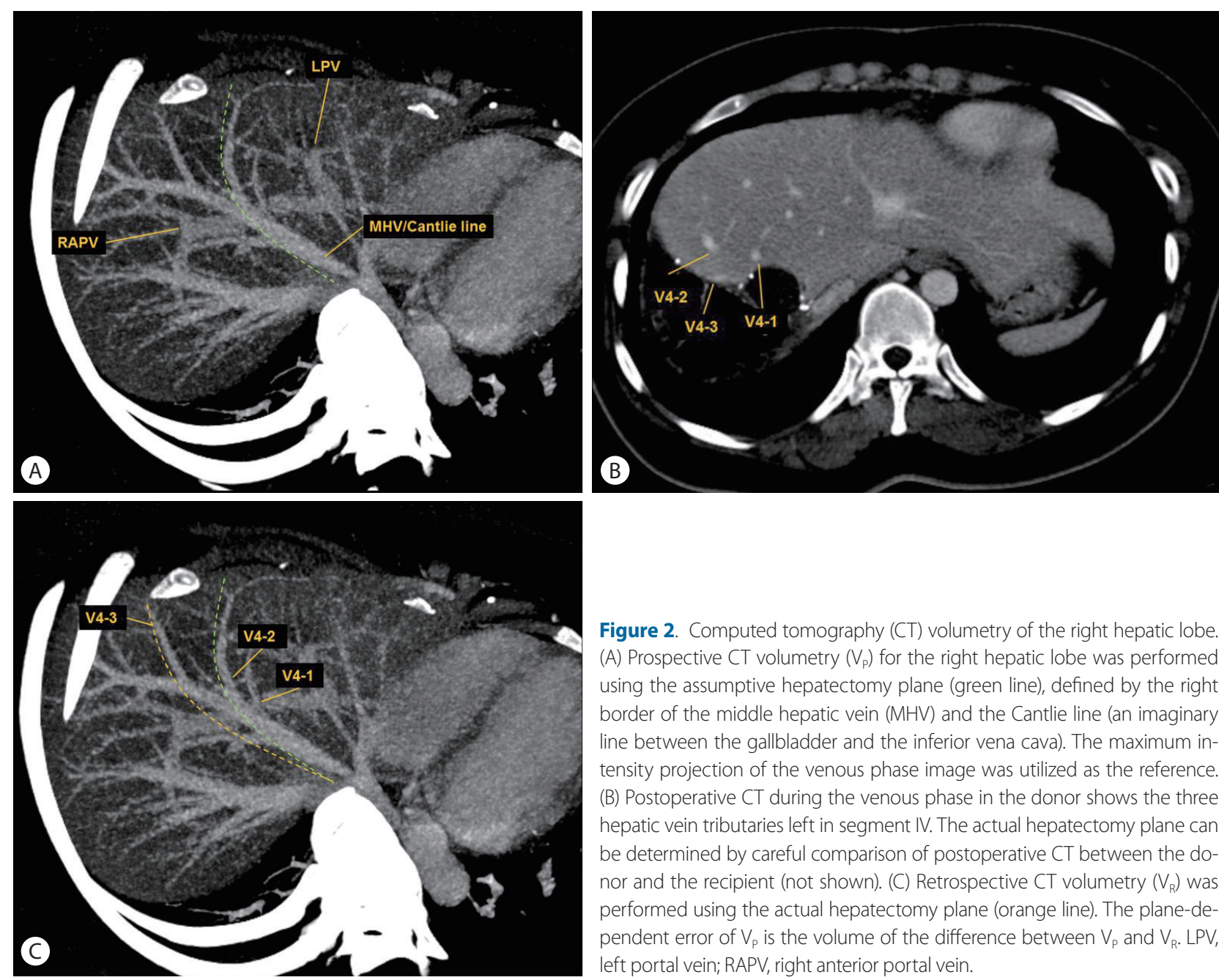

Figure 2. Computed tomography (CT) volumetry of the right hepatic lobe. (A) Prospective $\mathrm{CT}$ volumetry $\left(\mathrm{V}_{\mathrm{p}}\right)$ for the right hepatic lobe was performed using the assumptive hepatectomy plane (green line), defined by the right border of the middle hepatic vein (MHV) and the Cantlie line (an imaginary line between the gallbladder and the inferior vena cava). The maximum intensity projection of the venous phase image was utilized as the reference. (B) Postoperative $\mathrm{CT}$ during the venous phase in the donor shows the three hepatic vein tributaries left in segment IV. The actual hepatectomy plane can be determined by careful comparison of postoperative CT between the donor and the recipient (not shown). (C) Retrospective $C T$ volumetry $\left(V_{R}\right)$ was performed using the actual hepatectomy plane (orange line). The plane-dependent error of $V_{p}$ is the volume of the difference between $V_{p}$ and $V_{R}$. LPV, left portal vein; RAPV, right anterior portal vein.

Table 2. $V_{p}$ and $V_{R}$ deviation compared with $W$ of the right hepatic lobe in 46 living liver donors

\begin{tabular}{lcccc} 
& \multicolumn{1}{c}{ Mean \pm SD } & Median & $\mathbf{2 5}^{\text {th }}$ percentile & $\mathbf{7 5}^{\text {th }}$ percentile \\
\hline Error of $\mathrm{V}_{p}(\mathrm{~mL})$ & $73.3 \pm 52.4(57.7,88.8)$ & $69.2(37.0,89.2)$ & $28.1(18.9,41.5)$ & $107.8(83.5,127.8)$ \\
\hline Error of $\mathrm{V}_{R}(\mathrm{~mL})$ & $64.4 \pm 48.5(50.0,78.8)$ & $53.9(37.2,86.8)$ & $17.5(9.1,42.8)$ & $105.3(77.8,122.4)$ \\
\hline \% Error of $\mathrm{V}_{p}$ & $10.7 \pm 7.6(8.5,13.0)$ & $10.0(5.1,13.0)$ & $3.7(2.7,6.0)$ & $16.4(12.6,19.5)$ \\
\% Error of $\mathrm{V}_{R}$ & $9.3 \pm 6.7(7.3,11.3)$ & $9.0(5.8,12.6)$ & $2.7(1.2,6.1)$ & $15.6(12.5,17.6)$ \\
Plane-dependent error of $\mathrm{V}_{p}$ & $32.4 \pm 27.0(24.3,40.4)$ & $24.0(19.2,29.3)$ & $13.7(7.5,19.8)$ & $42.4(29.0,72.1)$ \\
\% Plane-dependent error of $\mathrm{V}_{p}$ & $4.7 \pm 4.0(3.5,5.9)$ & $3.3(2.7,4.1)$ & $2.0(1.3,2.8)$ & $6.7(3.9,9.3)$ \\
\hline
\end{tabular}

Numbers in parentheses are $95 \%$ confidence intervals

$\mathrm{CT}$, computed tomography; $\mathrm{V}_{\mathrm{Pr}}$ prospective $\mathrm{CT}$ volumetry; $\mathrm{V}_{\mathrm{R}}$, retrospective $\mathrm{CT}$ volumetry; $\mathrm{W}$, intraoperative weight; $\mathrm{SD}$, standard deviation.

Even with application of the actual resection plane, $V_{R}$ showed considerable difference $(9.3 \% \pm 6.7)$ from $W$ in our study. In $>80 \%$ of the subjects, $V_{R}$ was overestimated compared with W. Such a tendency of overestimation is consistent with that observed in previous studies. ${ }^{9,12,16,24,25}$ The blood volume circulating in the large hepatic vessels is considered to be the major cause of overestimation of CT volumetry. ${ }^{26}$ It has been shown that the blood pool was $>20 \%$ of the whole liver volume in one animal study. ${ }^{10}$ Similarly, Hwang et al. ${ }^{11}$ also suggested that blood-filled grafts contain approximately $29 \mathrm{~mL}$ of blood per $100 \mathrm{~g}$. Therefore, this kind of error may be compensated for by applying a conversion factor between the graft volume and weight ${ }^{12,27}$ or by using 
Heon-Ju Kwon, et al. Resection plane-dependent error of CT volumetry

computer-aided liver volumetry that can assess the bloodless liver volume. ${ }^{9}$ Another possible cause of error in $V_{R}$ may be the variable physiological density of the hepatic graft, but the effect of physical density on the accuracy of CT volumetry must be further evaluated.

There are several limitations to this study. First, we did not assess the reproducibility of $\mathrm{CT}$ volumetry between operators or at different time points in our study. Also, it was not verified whether the resection plane-dependent error of CT volumetry varied depending on the experience of the operator. Therefore, a further study should be followed to determine the inter- and intra-observer variation in the performance of this technique. Second, although we carefully compared preoperative and postoperative CT scans in donors and postoperative CT scans in recipients to determine the actual resection plane, over or insufficient correction might have been possible, as the margin of the area of the right and left hemiliver may undulate or even interdigitate within the liver. We utilized the middle hepatic vein and the Cantlie line to divide the right and left hemiliver, but currently there are several commercially available softwares that provide the division of the right and left hemiliver based on the portal territory. While it is anticipated that resection plane-dependent error may decrease with this algorithm for liver division, the performance of these softwares should be analysed by further well-designed study.

In conclusion, CT volumetry in the prospective setting may produce about a $5 \%$ error, attributed to surgical plane mis-registration. The plane-dependent error of $V_{p}$ exceeded $10 \%$ of $W$ in approximately $10 \%$ of the subjects in our study. This amount of error should be considered in the prospective setting especially when CT volumetry is performed by a less experienced operator unacquainted with the donor hepatectomy plane.

\section{Authors' contribution}

Dr. Kwon and Dr. KW Kim have full access to all of the data in the study and take responsibility for the integrity of the data and the accuracy of the data analysis.

- Study concept and design: Kwon and KW Kim.

- Acquisition of data: CS Lee, BH Kim, SY Kim, Song, and SG Lee.

- Analysis and interpretation of data: Kwon and JG Lee.

- Drafting of the manuscript: Kwon and KW Kim.

- Critical revision of the manuscript for important intellectual content: KW Kim, SY Kim, and SG Lee.

- Statistical analysis: Kwon, BH Kim, Song, and JG Lee.

\section{Acknowledgements}

This research was supported by the Basic Science Research Program of the National Research Foundation of Korea, funded by the Ministry of Education, Science, and Technology (grant 20100021107).

\section{Conflicts of Interest}

The authors have no conflicts to disclose.

\section{REFERENCES}

1. Lee SG, Park KM, Lee YJ, Hwang S, Choi DR, Ahn CS, et al. 157 adult-to-adult living donor liver transplantation. Transplant Proc 2001;33:1323-1325.

2. Inomata Y, Uemoto S, Asonuma K, Egawa H. Right lobe graft in living donor liver transplantation. Transplantation 2000;69:258-264.

3. Suh K, Lee K, Roh HR, Koh YT, Minn KW, Kim SJ, et al. Outcome of adult living donor liver transplantation using small volume of left liver graft less than 1\% of body weight. Transplant Proc 2001;33:14011402.

4. Kiuchi T, Tanaka K, Ito T, Oike F, Ogura Y, Fujimoto Y, et al. Smallfor-size graft in living donor liver transplantation: how far should we go? Liver Transpl 2003;9:S29-S35.

5. Ito T, Kiuchi T, Egawa H, Kaihara S, Oike F, Ogura Y, et al. Surgeryrelated morbidity in living donors of right-lobe liver graft: lessons from the first 200 cases. Transplantation 2003;76:158-163.

6. Fan ST, Lo CM, Liu CL, Yong BH, Chan JK, Ng IO. Safety of donors in live donor liver transplantation using right lobe grafts. Arch Surg 2000;135:336-340.

7. Lee SG, Hwang S. How I do it: assessment of hepatic functional reserve for indication of hepatic resection. J Hepatobiliary Pancreat Surg 2005;12:38-43.

8. Hwang S, Lee SG, Lee YJ, Sung KB, Park KM, Kim KH, et al. Lessons learned from 1,000 living donor liver transplantations in a single center: how to make living donations safe. Liver Transpl 2006;12:920-927.

9. Kim KW, Lee J, Lee H, Jeong WK, Won HJ, Shin YM, et al. Right lobe estimated blood-free weight for living donor liver transplantation: accuracy of automated blood-free CT volumetry--preliminary results. Radiology 2010;256:433-440.

10. Müller SA, Bläuer K, Kremer M, Thorn M, Mehrabi A, Meinzer HP, et al. Exact CT-based liver volume calculation including nonmetabolic liver tissue in three-dimensional liver reconstruction. J Surg Res 2010;160:236-243.

11. Hwang S, Lee SG, Kim KH, Park KM, Ahn CS, Moon DB, et al. Correlation of blood-free graft weight and volumetric graft volume by an analysis of blood content in living donor liver grafts. Transplant Proc 
2002:34:3293-3294.

12. Lemke AJ, Brinkmann MJ, Schott T, Niehues SM, Settmacher $U$,

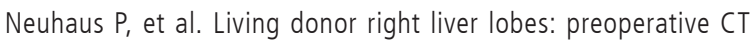
volumetric measurement for calculation of intraoperative weight and volume. Radiology 2006;240:736-742.

13. Karlo C, Reiner CS, Stolzmann P, Breitenstein S, Marincek B, Weishaupt $D$, et al. CT- and MRI-based volumetry of resected liver specimen: comparison to intraoperative volume and weight measurements and calculation of conversion factors. Eur J Radiol 2010;75:e107-e111.

14. Heymsfield SB, Fulenwider $T$, Nordlinger B, Barlow $R$, Sones $P$, Kutner M. Accurate measurement of liver, kidney, and spleen volume and mass by computerized axial tomography. Ann Intern Med 1979;90:185-187.

15. Hwang S, Lee SG, Ha TY, Song GW, Kim DS, Jung JP, et al. Tailoring transection of segment $V$ vein for optimal sharing of middle hepatic vein in right-lobe living donor liver transplantation. Hepatogastroenterology 2006;53:904-908.

16. Kamel IR, Kruskal JB, Warmbrand G, Goldberg SN, Pomfret EA, Raptopoulos V. Accuracy of volumetric measurements after virtual right hepatectomy in potential donors undergoing living adult liver transplantation. AJR Am J Roentgenol 2001;176:483-487.

17. Frericks BB, Kiene T, Stamm G, Shin H, Galanski M. CT-based liver volumetry in a porcine model: impact on clinical volumetry prior to living donated liver transplantation. Rofo 2004;176:252-257.

18. Emiroglu R, Coskun M, Yilmaz U, Sevmis S, Ozcay F, Haberal M. Safety of multidetector computed tomography in calculating liver volume for living-donor liver transplantation. Transplant Proc 2006;38:3576-3578.

19. Hiroshige S, Shimada M, Harada N, Shiotani S, Ninomiya M,
Minagawa $R$, et al. Accurate preoperative estimation of liver-graft volumetry using three-dimensional computed tomography. Transplantation 2003;75:1561-1564.

20. Radtke A, Sotiropoulos GC, Nadalin S, Molmenti EP, Schroeder T, Lang $\mathrm{H}$, et al. Preoperative volume prediction in adult living donor liver transplantation: how much can we rely on it? Am J Transplant 2007;7:672-679.

21. Lee J, Kim N, Lee $H$, Seo JB, Won HJ, Shin YM, et al. Efficient liver segmentation using a level-set method with optimal detection of the initial liver boundary from level-set speed images. Comput Methods Programs Biomed 2007;88:26-38.

22. Malladi R, Sethian JA, Vemuri BC. Shape modeling with front propagation: a level set approach. IEEE Trans Pattern Anal Mach Intell 1995;17:158-175.

23. Strunk H, Stuckmann G, Textor J, Willinek W. Limitations and pitfalls of Couinaud's segmentation of the liver in transaxial Imaging. Eur Radiol 2003;13:2472-2482.

24. Nakayama Y, Li Q, Katsuragawa S, Ikeda R, Hiai Y, Awai K, et al. Automated hepatic volumetry for living related liver transplantation at multisection CT. Radiology 2006;240:743-748.

25. Satou S, Sugawara Y, Tamura S, Yamashiki N, Kaneko J, Aoki T, et al. Discrepancy between estimated and actual weight of partial liver graft from living donors. J Hepatobiliary Pancreat Sci 2011;18:586-591.

26. Salvalaggio PR, Baker TB, Koffron AJ, Fryer JP, Clark L, Superina RA, et al. Liver graft volume estimation in 100 living donors: measure twice, cut once. Transplantation 2005;80:1181-1185.

27. Yoneyama T, Asonuma K, Okajima H, Lee KJ, Yamamoto H, Takeichi T, et al. Coefficient factor for graft weight estimation from preoperative computed tomography volumetry in living donor liver transplantation. Liver Transpl 2011;17:369-372. 